\title{
Verwendung von IMF-Schrauben zur mandibulo-maxillären Fixation
}

\author{
Andreas Hoffmann, Gerson Mast, Michael Ehrenfeld
}

\section{Zusammenfassung}

Im Vergleich zum Goldstandard der Okklusionssicherung, der Kieferbruchschiene, hat die IMFSchraube einige Vorteile (verkürzte Operationsdauer, verringerte Verletzungsgefahr für den Operateur, Verwendung vertrauter Techniken und Instrumente). Bei speziellen Indikationen, wie zum Beispiel bei Traumapatienten mit auf dem Blutweg übertragbaren Krankheiten, ist die Anwendung der IMF-Schrauben sicher eine Verbesserung. Nachteilig ist jedoch der begrenzte Anwendungsbereich (unkomplizierte Frakturen, kurzzeitige Liegedauer, keine begleitenden Zahnverletzungen). Eine Erweiterung des Indikationsspektrums erfordert eine Schraubenpositionierung an Stellen, die im Unterkiefer ein erhöhtes Risiko von Zahnwurzelverletzungen beinhalten. Dabei heilen wahrscheinlich Verletzungen der peripheren Zahnwurzel folgenlos ab. Sobald jedoch der Wurzelkanal eines Zahnes eröffnet wird, ist mit Folgeschäden zu rechnen. Entsprechend ist die Therapie mit IMF-Schrauben der mit Kieferbruchschiene nur bei wenigen Indikationen überlegen, und die IMF-Schrauben können wegen des geringen Indikationsbereiches die Therapie mit Kieferbruchschienen nicht ersetzen, sondern nur ergänzen.

\section{Einleitung}

Die Notwendigkeit der Okklusionssicherung durch eine mandibulo-maxilläre Fixation vor einer Osteosynthese in der Traumatologie des Gesichtsschädels und bei kieferorthopädischen Operationen wird heute allgemein anerkannt.

Mit der Verwendung von Kieferbruchschienen ist das Problem der zuverlässigen Okklusionssicherung gelöst [1]. Als verbesserbar gilt jedoch der Tragekomfort für den Patienten und das hohe Verletzungsrisiko für den Chirurgen.
Zur Reduzierung des Risikos werden neben den bekannten Regeln der Hygiene auch weitergehende Maßnahmen, wie z.B. die Verwendung doppelter Handschuhe, empfohlen [2]. Trotzdem ist gegenwärtig der Schutz des Operateurs vor einer Verletzung durch die scharfkantigen Kieferbruchschienen und die Drahtenden nicht zufriedenstellend. Vor diesem Hintergrund wurden die ersten Berichte über die Verwendung von selbstschneidenden Schrauben zur mandibulo-maxillären Fixation sehr positiv aufgenommen [3 -8]. Das in der Osteosynthese bewährte Prinzip einer im Knochen verankerten Schraube wurde hierfür abge-

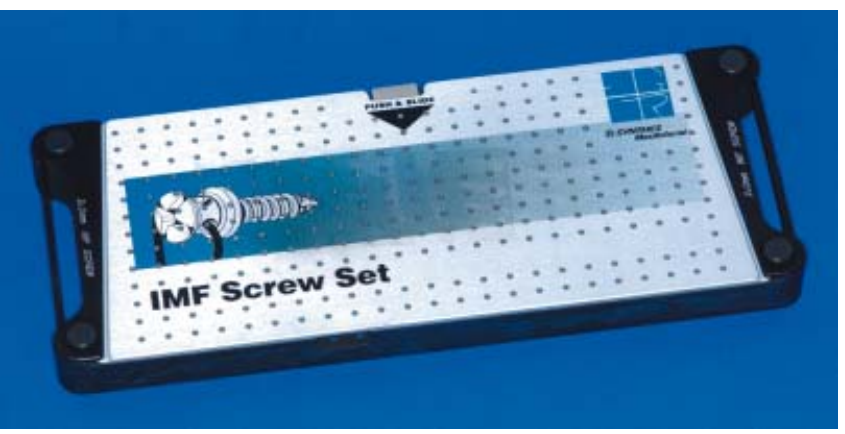

Abb. 1 Das Instrumentenmodul für IMF-Schrauben.

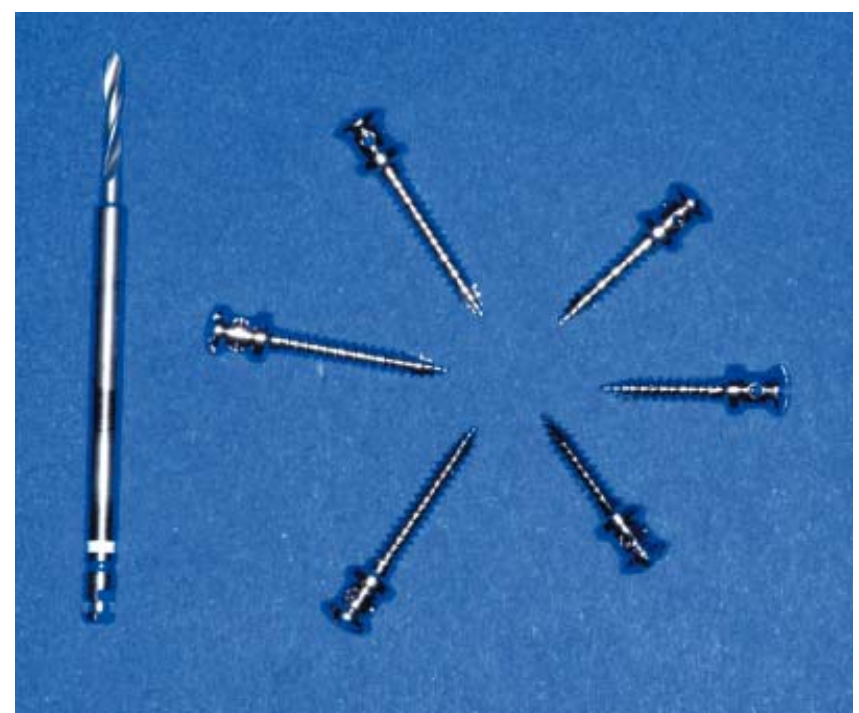

Abb. 2 IMF-Schrauben von 8 und $12 \mathrm{~mm}$ Länge mit einem Vorbohrer $(1,5 \mathrm{~mm})$.

OP-JOURNAL 2003; 19: 70-75

(C) Georg Thieme Verlag Stuttgart . New York 

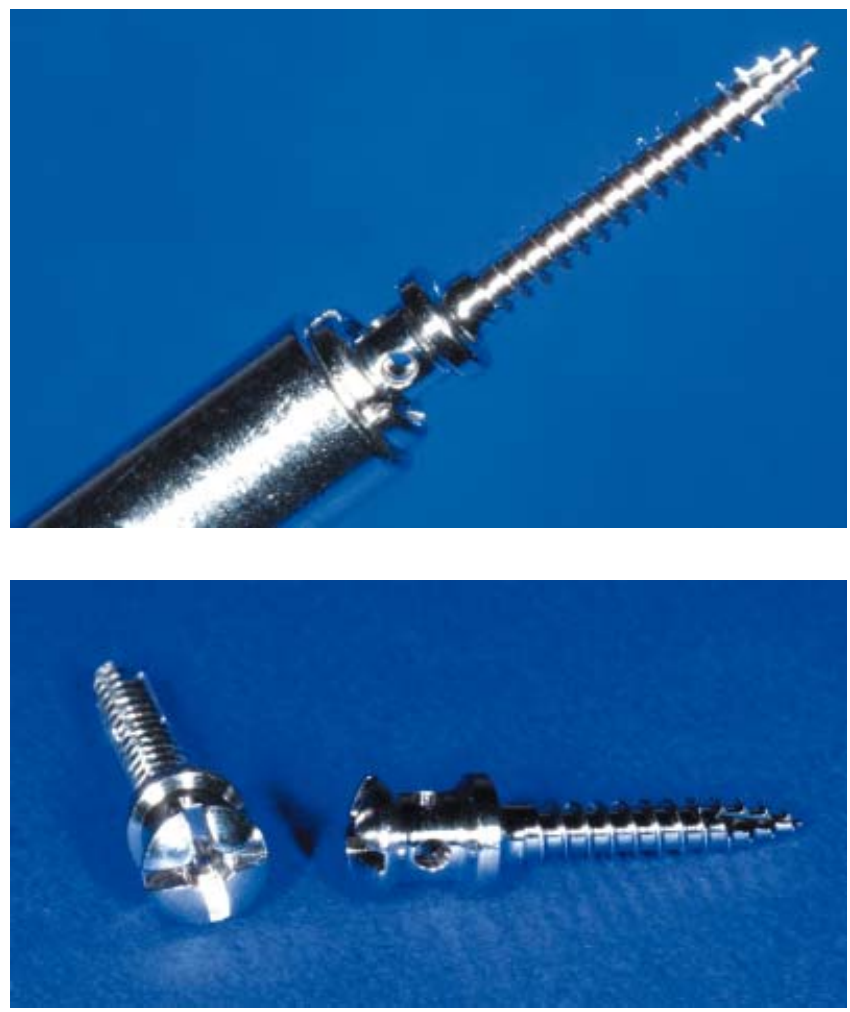

Abb. 3 IMF-Schraube durch die Haltehülse im Schraubendreher fixiert.

Abb. 4 Details des Schraubenkopfes zur Darstellung des Kreuzschlitzes sowie der Rille und der Perforationen zur Aufnahme der Drahtligaturen. wandelt und liefert dem Chirurgen eine neue Methode der mandibulo-maxillären Fixation mit vertrauten Arbeitsgängen und bekanntem Instrumentarium. Von der Arbeitsgemeinschaft für Osteosynthesefragen wurde eine selbstbohrende und selbstschneidende Stahlschraube entwickelt (IMF-Schraube), die zur temporären perioperativen Stabilisierung der Okklusion beim Erwachsenen empfohlen wird (Abb.1). Die Schraube hat einen Durchmesser von $2,0 \mathrm{~mm}$ und wird in 8 und $12 \mathrm{~mm}$ Länge geliefert (Abb. 2 u. 3). Im Schraubenkopf befindet sich eine Rille zur Sicherung der Drahtligaturen und parallel zum Kreuzschlitz verlaufende Bohrungen, falls der Draht eingefädelt werden soll (Abb.4). Als Indikation werden einfache, nicht dislozierte Frakturen des Ober- und Unterkiefers und kieferorthopädische Eingriffe angegeben. In einer vorläufigen klinischen Studie sollte nun die Eignung der IMF-Schraube (Fa. Stratec Medical, CH-4436 Oberdorf) im klinischen Alltag getestet werden.

\section{Material und Methode}

\section{Indikation}

Bei insgesamt 12 Patienten wurden IMFSchrauben zur temporären bzw. intraoperativen mandibulo-maxillären Fixation verwendet. Die Schrauben wurden

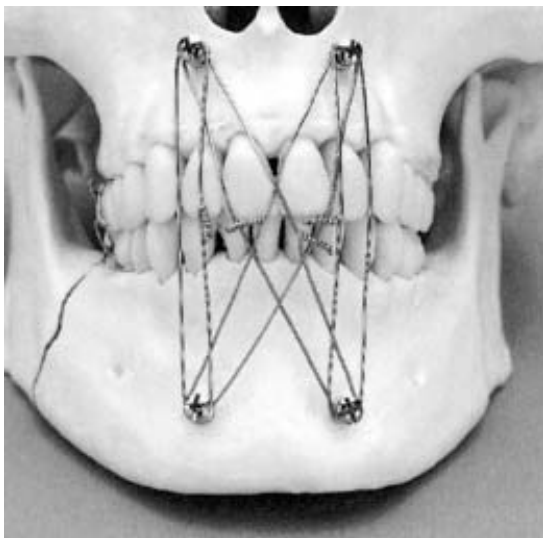

Abb.5 Vom Hersteller empfohlene Position der Schrauben und Verlauf der Drahtligaturen.

\section{Operationstechnik}

Der Hersteller empfiehlt eine Platzierung von insgesamt vier Schrauben, die oberhalb der maxillären bzw. unterhalb der mandibulären Zahnwurzeln medial oder lateral der Längsachsen der Eckzähne liegen. Zur Okklusionssicherung sollten die Drahtligaturen vertikal und über Kreuz angebracht werden (Abb.5).

jeweils zur Okklusionssicherung vor einer geplanten Osteosynthese des Ober- und Unterkiefers in Intubationsnarkose eingesetzt. Es handelte sich bei drei Patienten um eine Oberkiefervorverlagerung, bei einem Patienten um die Versorgung einer Oberkieferfraktur und bei 8 Patienten um operative Versorgungen von Unterkieferfrakturen. Dabei lagen fünf einfache Unterkieferfrakturen (2 tiefe dislozierte Kollumfrakturen, 2 Kieferwinkelfrakturen und 1 Medianfraktur) und drei doppelte Unterkieferfrakturen (Korpus- mit Kollumfraktur, Medianmit Kieferwinkelfraktur und Korpus- mit Kieferwinkelfraktur) vor.
In der vorliegenden Studie positionierten wir die Schrauben anders als vom Hersteller empfohlen, da mit der angegebenen Methode die Okklusionssicherung, v.a. im Seitenzahngebiet, nicht ausreichend war. Die Schrauben wurden an folgenden Stellen eingebracht:

1. Als Standardtechnik $(n=7)$ wurden pro Patient insgesamt 6 IMF-Schrauben zur Okklusionssicherung verwendet. Dabei wurden interdental im Ober- und Unterkiefer korrespondierende IMF-Schrauben median sowie beidseits im Seitenzahnbereich inseriert.

2. Bei zwei Patienten wurde zunächst im Oberkiefer eine individuelle Kiefer-

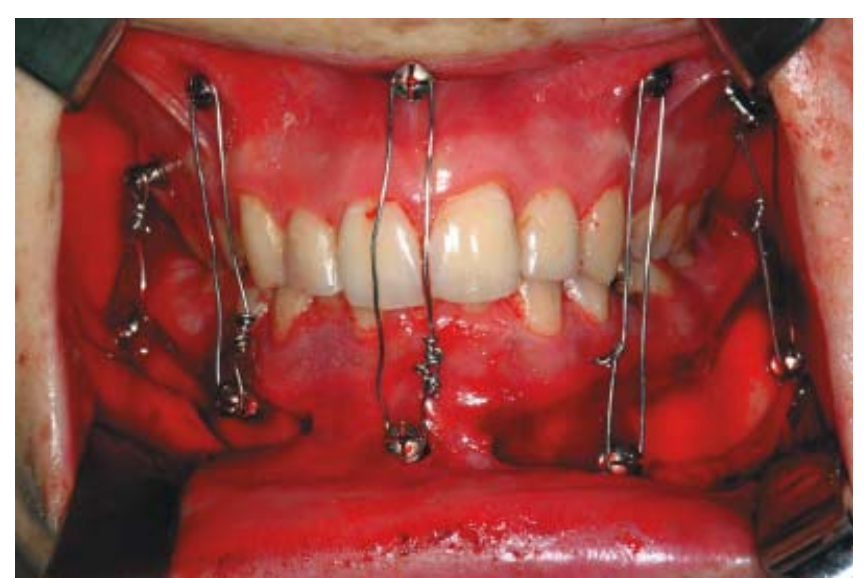

Abb. 6 Verbesserung der Okklusionssicherung, v. a. im distalen Bereich, durch eine MehrPunkt-Fixation. 
bruchschiene einligiert und dann, aufgrund eines ausgeprägten Tiefbisses, im Unterkiefer IMF-Schrauben verwendet (3 bzw. 5 Schrauben).

3. Bei einem Patienten wurden im Oberund Unterkiefer jeweils 5 Schrauben verwendet. Diese wurden interdental median sowie beidseits auf Höhe der Eckzähne und im Seitenzahnbereich inseriert (Abb.6).

4. Bei zwei Patienten wurde je eine Schraube median oben und unten verwendet.
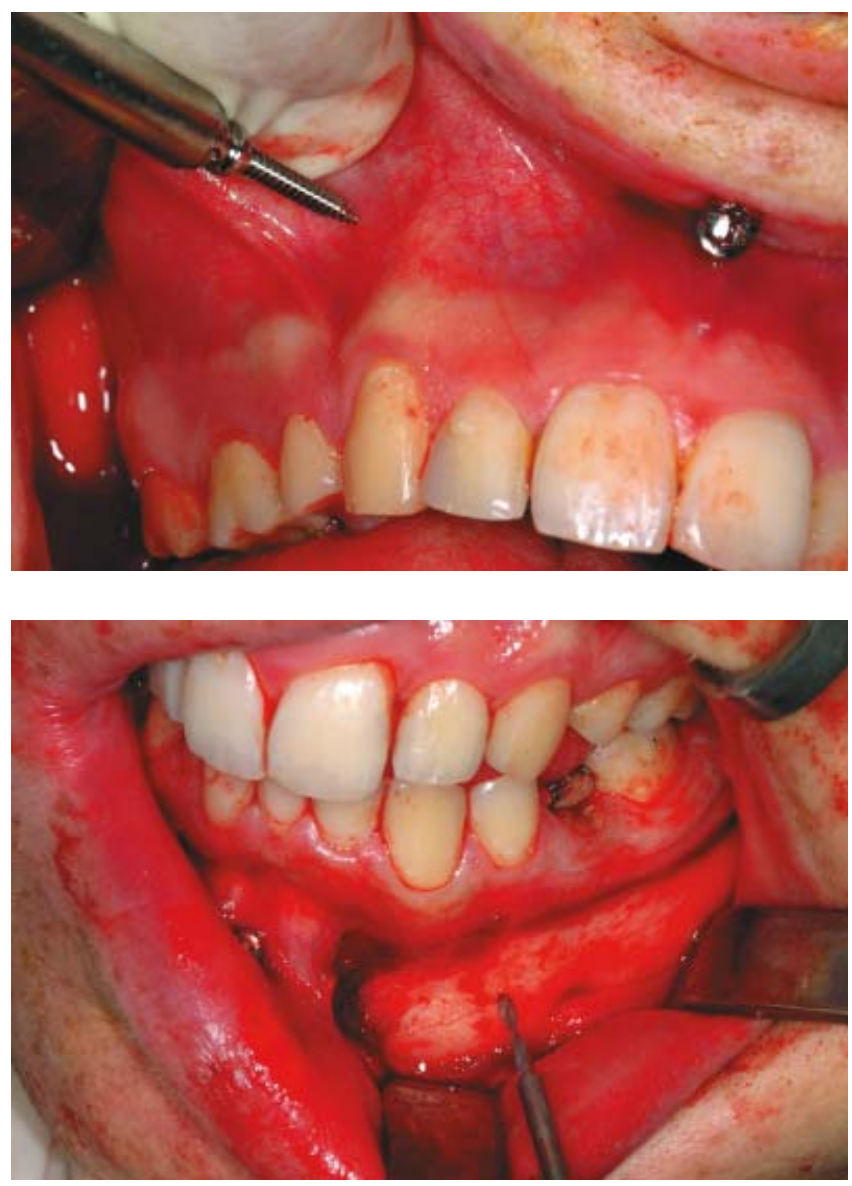
über den Verlauf der

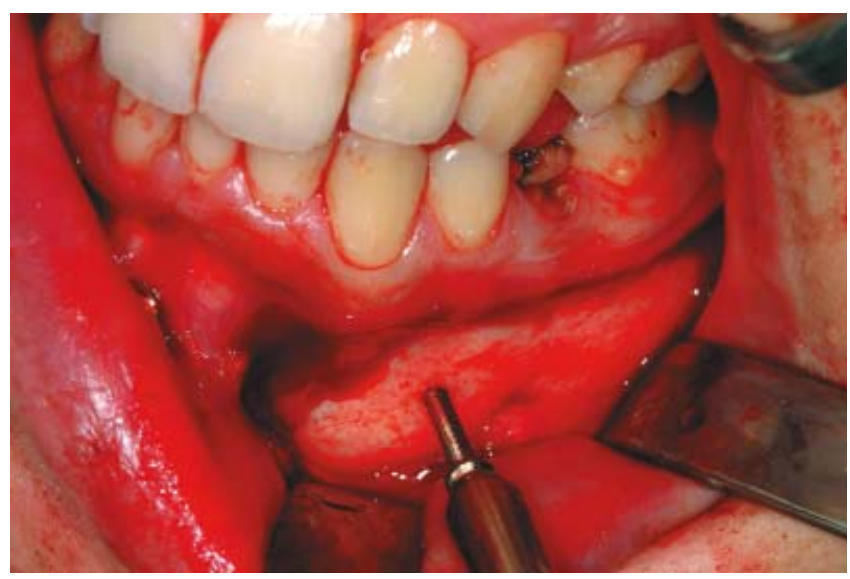

Abb. 7 Überprüfen des Verlaufes der Eckzahnwurzel durch Palpation und transmuköses Einbringen der Schrauben ohne Vorbohrung.

Abb. 8 Zur sicheren Schonung wichtiger anatomischer Strukturen (N. mentalis) und zur besseren Orientierung Seitenzahnwurzeln wird die Außenfläche des Unterkiefers dargestellt und ein Loch vorgebohrt.

\section{Verweildauer der Schrauben}

Bei der Mehrzahl der Patienten $(\mathrm{n}=9)$ wurden die Schrauben nach erfolgter Osteosynthese intraoperativ wieder entfernt. Bei einem Patienten entfernten wir die Schrauben am 10., bei zwei Patienten am 20. postoperativen Tag. Die Schrauben konnten ohne Anästhesie entfernt werden, wenn der Schraubenkopf nicht vom umgebenden Weichgewebe überwuchert wurde.

\section{Nachuntersuchung}

Postoperativ wurden bei allen Patienten zur Kontrolle des Repositionsergebnisses ein Orthopantomogramm und eine Schädelaufnahme in okzipito-frontalem Strahlengang durchgeführt, bei zwei Patienten verwendeten wir ergänzend Zahnfilme. Die klinische Nachuntersuchungszeit betrug mindestens vier Wochen und maximal 12 Monate.

\section{Ergebnisse}

\section{Okklusionssicherung}

Bei allen Patienten gelang die Einstellung der Okklusion über die IMF-Schrauben problemlos. Dabei war eine Fixation über je drei Schrauben im Ober- und Unterkiefer für die gewünschte Stabilität ausreichend.

Im Frontzahnbereich genügte die Rille im Schraubenkopf zur Fixierung der Drahtligatur. Im Seitenzahngebiet waren die Achsen der korrespondierenden Schrauben häufig konvergent, so dass der Unterschnitt des Schraubenkopfes für die Fixation der Drahtligaturen nicht ausreichte und zumindest an einer Schraube der Draht durch die Bohrung im Kopf geführt werden musste. Jeweils 2 Schrauben im Ober- und 2 Schrauben im Unterkiefer konnten nach Insertion wegen der fehlenden Stabilität primär nicht verwendet werden und mussten zweimal gesetzt werden.

Aber Abschieben des Mukoperiosts und Vorbohrung.

\section{Verletzungen von Zahnwurzeln}

In der postoperativen Röntgenkontrolle zeigten sich bei sechs der zwölf Patienten Verletzungen der Zahnwurzeln durch IMF-Schrauben. Es handelte sich um drei Front- und fünf Seitenzähne des Unterkiefers. Zwei Zähne waren wurzelgefüllt und sechs Zähne präoperativ vital (Abb.10). Fünf Zähne wurden zentral verletzt, d.h. die Bohrung erreichte den Wurzelkanal (Abb.12). Bei drei Zähnen lag der 


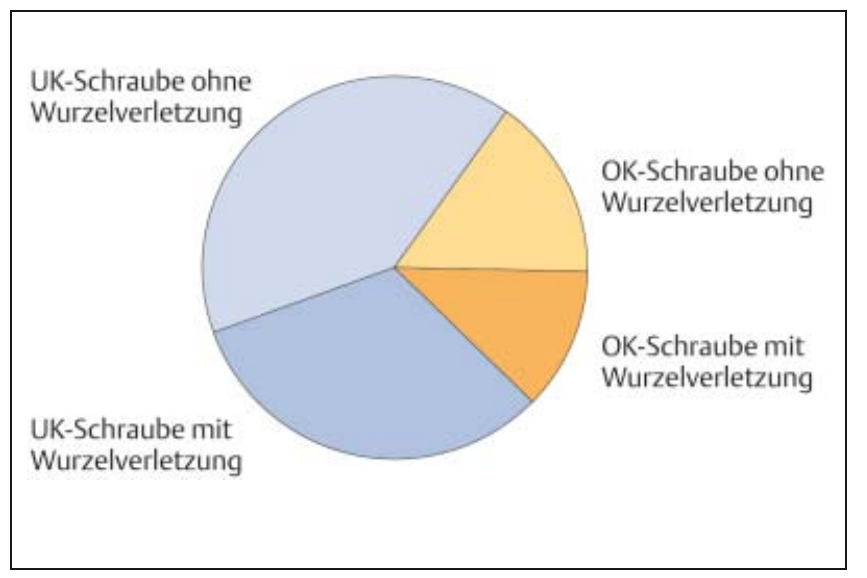

Abb.10 Anteil der eine Zahnverletzung (zentral und peripher) verursachenden IMF-Schrauben an der Gesamtmenge $(n=62)$.
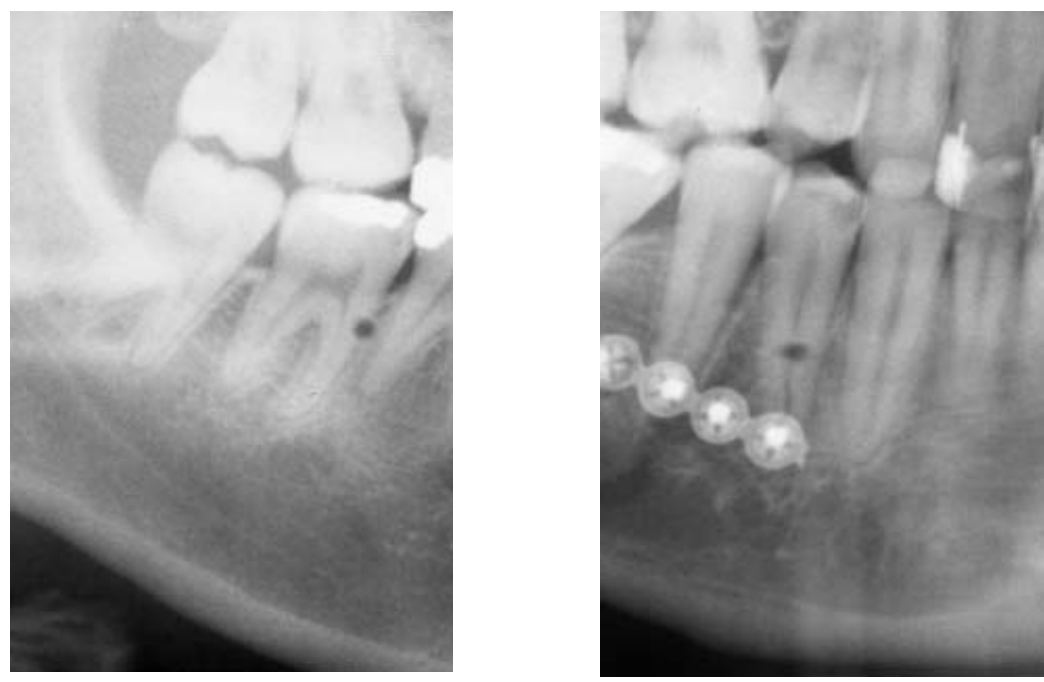

Abb.11 Periphere Verletzung der Wurzel des Zahnes 47, welche wahrscheinlich folgenlos abheilen wird.
Abb.12 Zentrale Verletzung der Wurzel des Zahnes 44 - eine Eröffnung des Wurzelkanals ist anzunehmen.
Defekt peripher, d.h. im Bereich des Parodontalspaltes (Abb.11).

Als Langzeitkomplikation musste ein Seitenzahn extrahiert werden, nachdem er zehn Wochen nach einer zentralen Verletzung durch eine IMF-Schraube frakturierte. Einmal wurde an einem Seitenzahn eine Wurzelspitzenresektion notwendig, da sich ebenfalls nach einer zentralen Wurzelverletzung eine Infektion der Wurzelspitze zeigte (Abb.13 u. 14).

Ein Absterben der übrigen verletzten Zähne wurde bisher nicht beobachtet.

\section{Reaktionen der Weichteile}

Bei den intraoperativ bzw. nach 10 Tagen entfernten Schrauben zeigte sich keinerlei Reaktion der umgebenden Weichgewebe. Die Schrauben traten jeweils im Bereich der mukogingivalen Grenze durch die Schleimhaut. Bei beiden Patienten mit einer Liegedauer der Schrauben von 20 Tagen zeigte sich betont im unteren Vestibulum eine deutliche Granulationsgewebsbildung in der Umgebung der Schraubenköpfe. Die Patienten tolerierten dabei die Schrauben schlecht, da beispielsweise durch jede Bewegung der Unterlippe Schmerzen ausgelöst wurden. Die Schrauben sowie das Granulationsgewebe wurden in terminaler Anästhesie entfernt. Die Schrauben waren jeweils im Bereich der beweglichen Mundschleimhaut inseriert.

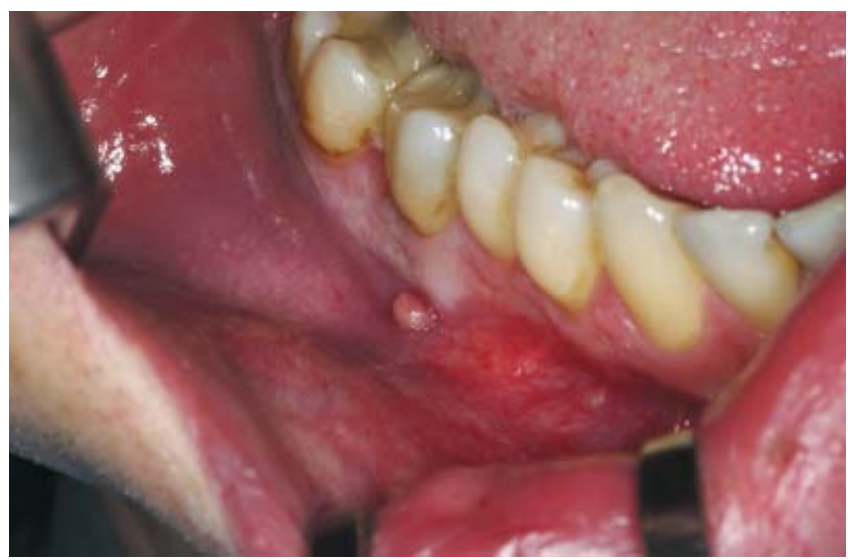

Abb.13 Vestibuläre Fistel als Folge einer Verletzung des wurzelgefüllten Zahnes 46.

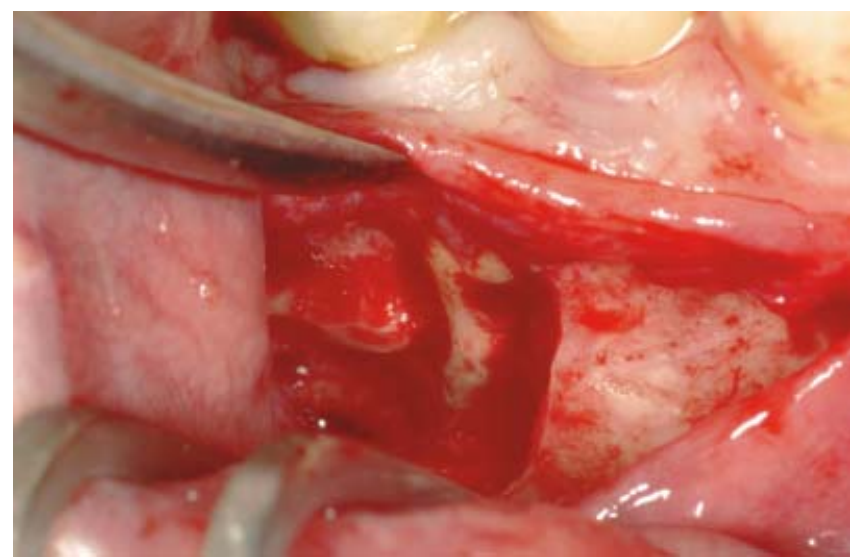

Abb.14 Nach Exzision des Fistelmauls, Abschieben des Mukoperiosts und bukkaler Ostektomie zeigt sich der Defekt im Bereich der mesialen Wurzel des Zahnes 46. 


\section{Diskussion}

Eine Neuentwicklung zur Okklusionssicherung durch eine mandibulo-maxilläre Fixation muss sich am bisherigen Goldstandard, der Kieferbruchschiene, messen lassen.

In den Anwendungsrichtlinien der IMFSchrauben wird eine Fixation über insgesamt vier Schrauben angegeben, die jeweils apikal der Wurzeln der Eckzähne inseriert werden sollten. Die Indikation ist auf einfache Unterkieferfrakturen ohne Dislokation beschränkt. Wir verwendeten die Schrauben zunächst entsprechend den Richtlinien. Dabei zeigte sich jedoch, dass so die Sicherung der Okklusion im Seitenzahngebiet oft ungenügend war. Der Hersteller beschreibt zwar, dass durch eine Positionierung der Schrauben lateral der Eckzahnachse die Okklusionskontrolle v.a. im Seitenzahngebiet verbessert wird, was wir jedoch nicht bestätigen konnten. Ein weiterer Schwachpunkt der empfohlenen Technik ist die große Entfernung der Schrauben von der Okklusionsebene. Das Anziehen der Drahtligatur wirkt als exzentrische Kraft auf die Fragmente und erschwert die Reposition vor allem am Unterrand des Unterkiefers. Bei protrudierten Frontzähnen laufen die über Kreuz liegenden Drahtligaturen zudem über die Kanten der Zahnkronen und können dadurch ein sicheres Anziehen der Ligaturen verhindern bzw. eine feste Ligatur kann über die Zahnkrone abgleiten und sich unbemerkt lockern. Zudem traumatisiert die apikale Positionierung bei längerer Liegedauer der Schrauben die Weichteile deutlich, da die Schrauben in der beweglichen Schleimhaut zu liegen kommen.

Aufgrund dieser Probleme entschieden wir uns, die Schrauben näher an der Okklusionsebene zu platzieren und zumindest über eine 3-Punkt-Abstützung durch insgesamt 6 Schrauben die Okklusionssicherung zu verbessern.

Mit dieser 3-Punkt-Abstützung gelang in allen Fällen eine zügige und verlässliche manibulo-maxilläre Fixation.

Die Verletzungsgefahr für den Chirurgen ist bei der Insertion der Schrauben minimal. Es bleibt jedoch ein Restrisiko von Stichverletzungen durch die Drahtenden. Dieses kann nur durch die Anwendung von Gummizügen anstelle von Drahtligaturen vermieden werden. Bei gleicher Zuverlässigkeit der Okklusionssicherung ist die Methode schneller als die Therapie mit konventionellen Schienenverbänden. Dieser Zeitvorteil kann sich jedoch relativieren, wenn Schrauben sich lockern und neu fixiert werden müssen, was insbesondere im Oberkieferseitenzahnbereich bei einer dünnen Kieferhöhlenwand auftreten kann. Verzögernd wirkt auch, wenn die Längsachsen der korrespondierenden Schrauben nicht ausreichend parallel zueinander sind. Daraus resultiert eine konvergente Stellung der Schraubenköpfe und der Unterschnitt im Schraubenkopf ist nicht mehr ausreichend, um ein Abgleiten des Drahtes zu verhindern - der Draht muss durch die Perforationen im Schraubenkopf geführt werden.

Über den langfristigen Tragekomfort für die Patienten können wir keine suffiziente Aussage machen, da nur bei $3 \mathrm{~Pa}$ tienten die Schrauben für ein bzw. zwei Wochen nach der Operation belassen wurde. Bei den übrigen Patienten erfolgte die Schraubenentfernung am Ende der Operation. Die starke Granulationsgewebsbildung bei in der beweglichen Schleimhaut liegenden Schraubenköpfen und die damit einhergehenden Beschwerden der Patienten veranlassten uns, die IMF-Schrauben tendenziell schneller als Kieferbruchschienen zu entfernen. Damit vergibt man sich jedoch die Möglichkeit einer funktionellen Nachbehandlung, z.B. zur Verbesserung kleiner okklusaler Inkongruenzen.

Als häufigste Nebenwirkung zeigt sich bei der Therapie mit Kieferbruchschienen nach längerer Liegedauer eine Gingivitis, die vorübergehend ist und meist innerhalb weniger Tage nach Entfernung der Schienen folgenlos abheilt. In Einzelfällen kann eine gingivale Rezession resultieren, die jedoch selten mehr als $1-2 \mathrm{~mm}$ betragen dürfte. Dagegen sind die Zahnwurzelverletzungen durch die IMFSchrauben eine folgenreichere Schädigung. Key und Gibbons (2001) geben an, dass bleibende Schäden an einem Zahn, z.B. ein Absterben, nur auftreten, wenn die Schraube den Zahn mittig trifft und damit der Wurzelkanal eröffnet wird [7]. In unserer Studie wurden durch die insgesamt 62 verwendeten Schrauben 5 Zähne zentral verletzt. Bei einem Zahn führte dies zu einer Fraktur und er wurde entfernt. Bei einem Zahn wurde eine Wurzelspitzenresektion notwendig. Zwei der drei verbliebenen Zähne waren präoperativ vital, und es zeigte sich postoperativ bisher keine Devitalisierung der Zähne, was jedoch durch den kurzen Beobachtungszeitraum keine langfristige Verlaufsprognose zulässt.
Zur Vermeidung von Folgeschäden ist die Positionierung der Schrauben entscheidend [7]. Diese gelingt im Oberkiefer relativ problemlos, da der Verlauf der Wurzeln transmuskös gut zu ertasten ist. Entsprechend zeigten sich in unserer Studie keine Verletzung der Oberkieferzähne. Im Unterkiefer ist die dreidimensionale Lokalisation des Wurzelverlaufes sowohl klinisch als auch radiologisch schwierig, was dadurch verdeutlicht wird, dass ausschließlich Zähne des Unterkiefers verletzt wurden.

In den aktuell vorliegenden Erfahrungsberichten über die Verwendung gleichartiger Systeme schwanken die Angaben über die Anzahl der Zahnwurzelverletzungen stark. Diese Komplikation und ihre langfristigen Folgen ist gegenwärtig Gegenstand heftiger Diskussionen [4-9].

Eine breite Verwendung der IMF-Schrauben in der klinischen Routine wird durch die strenge Beschränkung der Indikation auf einfache, nicht dislozierte Frakturen verhindert und ist unserer Meinung nach nicht unbedingt gerechtfertigt. Vermehrt man die Anzahl der Fixationspunkte auf drei pro Kiefer, ist damit die Okklusion auch für eine Anwendung bei dislozierten und doppelten Unterkieferfrakturen ausreichend stabil fixiert. Dafür ist jedoch im Seitenzahngebiet des Unterkiefers eine interdentale Lage der Schrauben notwendig, da eine Position unterhalb der Zahnwurzeln durch den Canalis mandibulae verhindert wird.

Entsprechend wird die Erweiterung der Indikation mit einem erhöhten Risiko von dauerhaften Folgeschäden (Verletzungen der Unterkieferzähne) erkauft. Eine ähnliche Schwierigkeit entsteht wenn die Schrauben zur längerfristigen Okklusionssicherung, z.B. im Rahmen einer rein konservativen Frakturversorgung, verwendet werden sollen. Zur Vermeidung starker Weichteilreaktionen sollten die Schrauben im Übergangsbereich von befestigter zu beweglicher Schleimhaut platziert werden, was ebenfalls zu einer interdentalen Schraubenposition führt und bei eng beieinander liegenden Wurzeln diese verletzt werden können.

Problematisch ist auch die Verwendung der IMF-Schrauben bei gleichzeitig vorliegenden Zahnluxationen. Die gelockerten Zähne werden bei einer Therapie mit einer Kieferbruchschiene gleichzeitig mit stabilisiert, was natürlich mit der IMF-Schraube nicht möglich ist. Hier werden zusätzlich Schienenverbände notwendig, was wenig praktikabel erscheint. 


\section{Literatur}

1 Austermann K. Frakturen des Gesichtsschädels. In: Schwenzer, N. and M. Ehrenfeld, (Hrsg). Zahn-Mund-Kieferheilkunde, Bd. 2: Spezielle Chirurgie. Vol. 3. Stuttgart: Thieme, 2002; 275-366

2 Avery C, Taylor J, Johnson P. Double gloving and a system for identifying glove perforations in maxillofacial trauma surgery. Br J Oral Maxillofac Surg 1999; 37: 316-319

3 Busch R. Maxillomandibular fixation with intraoral cortical bone screws: a 2-year experience. Laryngoscope 1994; 104: $1048-$ 1050

${ }^{4}$ Farr D, Whear N. Intermaxillary fixation screws and tooth damage. $\mathrm{Br} J$ Oral Maxillofac Surg 2002; 40: 84-85
${ }^{5}$ Holmes S, Hutchinson I. Caution in use of bicortical intermaxillary fixation screws (letter). Br J Oral Maxillofac Surg 2000; 38: 574

${ }^{6}$ Jones D. The intermaxillary screw: a dedicated bicortical bone screw for temporary intermaxillary fixation. Br J Oral Maxillofac Surg 1999; 37: 115-116

7 Key S, Gibbsons A. Re: Care in the placement of bicortical intermaxillary fixation screws. Br J Oral Maxillofac Surg 2001; 39:484

8 Majumdar A, Brook I: Iatrogenic injury caused by intermaxillar fixation screws. $\mathrm{Br} \mathrm{J}$ Oral Maxillofac Surg 2002; 40: 84

${ }_{9}^{9}$ Maurer P, Syska E, Eckert A, Berginski M, Schubert J. Die FAMI-Schraube für die temporäre intermaxilläre Fixation. Mund Kiefer Gesichtschir 2002; 6: 362
Dr. med. Dr. med. dent. Andreas Hoffmann

Assistenzarzt

Dr. med. Dr. med. dent. Gerson Mast Leitender Oberarzt

Prof. Dr. med. Dr. med. dent. Michael Ehrenfeld

Klinikdirektor

Klinik und Poliklinik für Mund-, Kiefer- und Gesichtschirurgie der Ludwig-Maximilians-Universität München Lindwurmstraße 2a D-80337 München 